\title{
Bénit, André. Légendes, intrigues et médisances autour des « archidupes " Charlotte de Saxe- Cobourg-Gotha, princesse de Belgique ; Maximilien de Habsbourg, archiduc d'Autriche : Récits historique et fictionnel. Bruxelles : Peter Lang, 2020.

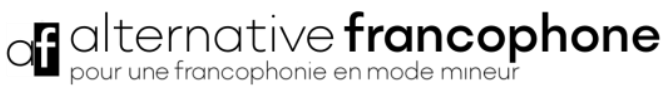

https://doi.org/10.1215/08992363-10.29173/af29426

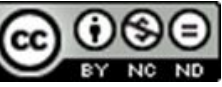

\section{Chris Reyns-Chikuma}

reynschi@ualberta.ca

University of Alberta

A ndré Bénit, professeur à la Autónoma (Madrid), spécialiste de littérature belge francophone, a déjà publié plusieurs articles et un livre sur la princesse Charlotte, fille de Léopold II, roi de

Belgique. Ce deuxième ouvrage est plus volumineux et plus ambitieux puisqu'il couvre la vie de la princesse et de son mari, l'archiduc d'Autriche, devenus empereur et impératrice du Mexique en 1866. Le premier intérêt de cette étude vient de la confrontation de la grande Histoire et de la petite histoire, la fiction historique (p. ex., le roman historique). Les deux sont bâtis sur des faits, mais dans ce cas, parce qu'il existe de nombreuses lacunes dans la connaissance de certains faits et de " nombreuses médisances », l'Histoire de ces deux souverains est incomplète et les fictions, très nombreuses, en profitent « pour pénétrer plus en profondeur l'âme de ses personnages, pour s'immiscer davantage dans leur intimité, dans leur vie privée, dans leur quotidienneté ; il [le biographe romancier] peut faire preuve de plus d'ironie et d'audace à tous les niveaux, notamment stylistique » (19).

Le livre comprend 6 chapitres qui correspondent aux étapes de la vie des deux personnages (1. Rencontre ; 2. Le Mexique ; 3. Miramar 1 : Château sur l'Adriatique ; 4. Miramar 2 ; 5 . La bataille de Querétaro et l'exécution de Maximilien ; 6 . Les 60 ans de «folie » de la princesse-impératrice). Chaque chapitre est divisé en 3 parties : la première, le récit historique ; la deuxième, le récit fictionnel, beaucoup plus long dans les trois cas, car citant de longs extraits de ces fictions ; la troisième, qui s'intitule " réflexions », est brève, mais essentielle pour résumer les problèmes qui ressortent de cette confrontation entre les deux 
Hhistoires qui d'ailleurs ne sont pas aussi facilement séparables que la division le fait croire (19). Bénit lui-même cite des romanciers dans la première partie historique et des historiens dans la seconde, fictionnelle. Même si Bénit semble faire un peu trop confiance aux historiens, comme il l'écrit à plusieurs reprises, ces historiens ont des perspectives parfois aussi problématiques dues à leurs propres convictions, et aux types de sources auxquelles ils avaient accès à leur époque comme le simple séjour de Charlotte à Paris en août 1866 le montre (229). De même, le passage d'un domaine à l'autre, comme Henriette Chandet, historienne devenue romancière, renforce cette porosité.

Je ne vais pas résumer chaque chapitre qui reprend de manière exhaustive les faits connus de ces deux vies. L'intérêt vient plutôt de la confrontation des suppositions très nombreuses et diverses sur les multiples faits inconnus ou méconnus. Bénit fait un travail tout à fait extraordinaire rassemblant et confrontant un grand nombre de sources (60 selon la bibliographie et donc plusieurs milliers de pages). Qu'il y ait tant d'historiens et d'écrivains qui s'intéressent à ces personnages, somme toute insignifiants puisqu'ils n'auront eu qu'un faible impact sur l'Histoire (chronologiquement quelques années, et historiquement ces deux personnages, déjà secondaires, n'ont pas changé grand-chose aux événements mondiaux ou même de leur pays (430). Ce type d'Histoire est aussi depuis longtemps déjà en perte de vitesse face à la « nouvelle histoire » qui s'occupe moins des « grands » de ce monde, de leurs événements officiels et de leurs batailles, et davantage des « wretched of the earth » (LeGoff et Nora) et dont des écrivain.e.s comme la romancière Blanche Coudurier se moquera «pour dénoncer les rêves de grandeur de ceux qui considèrent comme un dû les énormes privilèges dont ils bénéficient » (173). Les médias ont d'ailleurs pris le relais pour montrer les grandiloquences insolentes et les frasques révoltantes de ces familles royales, aujourd'hui encore, de la couronne britannique. ${ }^{1}$ Malheureusement, ces histoires sont souvent accueillies de manière peu critique par les «petits ». C'est aussi ces sentimentalisme, romantisme et sensationalisme qui laissent perplexes. Et ce livre témoigne de cette fascination souvent morbide, car à ces « légendes, intrigues et médisances », on pourrait ajouter «fake news », "alternative reality », et autres «théories du complot », comme première leçon de ce livre.

Toutefois, comme le titre l'indique et le sous-titre le confirme, le centre n'est pas vraiment les deux personnages ou ils n'en sont le centre qu'en tant qu' « archidupes ». Le jeu de mots « archiduc/archidupe » est tiré de l'ouvrage Charlotte et Maximilien. L'empire des archidupes (1993) de la journaliste belge Jeanine Lambotte. Bénit analyse les multiples facteurs, personnes et faits, qui entourent et influencent les deux personnages, moitié victime (sous des pressions multiples et énormes), moitié témoins, moitié bourreaux, et aussi moitié agent.e et acteur-actrice des intrigues, des médisances, des légendes et des échecs fracassants (voir le surnom de Maximilien, «l'empeorador = l'empireur », p. 172). C'est d'ailleurs évidemment un autre aspect de cette étude qui est intéressant puisqu'on se rend compte à la fois du nombre impressionnant de ces suppositions, mais aussi, grâce à la bonne contextualisation de Bénit, des motivations soit directes (intrigues, médisances), soit indirectes (besoin de légendes, mythes). Ces dernières sont cependant moins exploitées par Bénit, et le postfacier (connu dans le tout petit monde de la littérature belge ${ }^{2}$ ) en mentionne une sur Robert Goffin, historien et romancier belge qui publie un roman en 1937, Charlotte, l'impératrice fantôme, sur lequel il écrit :

\footnotetext{
${ }^{1}$ Par exemple, le Prince Charles a-t-il tenu des propos racistes sur l'enfant de Meghan ?

${ }^{2}$ Marc Quaghebeur, chercheur indépendant.
} 
En affirmant ainsi de façon péremptoire, que le général français Maxime Weygand, né à Bruxelles de parents inconnus, est le fils de l'impératrice Charlotte, ou en prétendant tout aussi crânement que l'empereur Maximilien est le fils de l'archiduchesse Sophie et du duc de Reichstadt, Robert Goffin peut faire de ce soldat, qui fut un acteur important de la victoire française de 1918, l'héritier qui eut dû succéder au vieil empereur François-Joseph en lieu et place de l'archiduc François-Ferdinand assassiné à Sarajevo ou de l'empereur Charles, dernier titulaire du trône d'Autriche-Hongrie. Voilà qui pourrait donner matière en outre à commentaires pour la fusion mythique des hantises habsbourgo-françaises - pour ne pas dire «bourguignonnes »-- d'une certaine imagination belgo-francophone. Quel superbe tremplin pour une lecture débridée de l'Histoire! Rien ne permet toutefois, jusqu'à nouvel ordre de l'attester (427).

Dans ce passage, au-delà des références aux faits historiques importants du passé (Sarajevo, où commença la boucherie de millions de «petits » au bénéfice de ces « grands ») et d'un passé bien complexe et révolu de rois, empereurs, ducs et autres consorts aux noms et titres longs et surannés, ce qui interpelle encore le démocrate et l'humaniste d'aujourd'hui, c'est combien ces mythes et légendes persistent, combien elles intéressent. Mais c'est aussi le deuxième degré de l'interprétation de ce postfacier des motivations profondes, mythiques, de ce « romancier-historien » et de ses lecteurs belgofrancophones, sur un passé glorieux qui a eu lieu (bourguignon) et un autre qui aurait pu avoir lieu (habsbourgo-français). En lisant l'étude de Bénit, on ne peut que se poser aussi ce genre de questions. Elle peut en effet se lire aussi au second degré sur au moins deux sujets qui ne sont que trop brièvement abordés et qui pourraient faire l'objet d'au moins deux autres ouvrages de la même ampleur : d'abord sur le roman historique et ensuite sur le point de vue alternatif, celui de l'Autre et concrètement celui des autres, non-Européens. Les deux sont présentés ici, mais insuffisamment et mériteraient un approfondissement dans deux autres ouvrages (à venir?).

Un autre ouvrage sur une comparaison plus technique (par ex. stylistique) des deux types de récits historiques, entre fictions et Histoire. Cette question a déjà été interrogée précédemment, par exemple, par Georg Lukacz, étudiant les grands romans historiques écrits pendant l'époque de ces empereurs, produits par les Walter Scott, Manzoni, Balzac, etc., plus tard Mann, et plus près de nous et dans une perspective du réalisme magique les Carpentier et les Marquez, etc., elle pourrait être prolongée théoriquement et contextuellement. La problématique est introduite dès les exergues par le biais du romancier belge Pierre Mertens et dans l'introduction avec Laurent Binet (dont le roman $H h H$ aussi superbe qu'énigmatique Himmlers Hirn heißt Heydrich - parle d'une Hhistoire qui concerne les humanistes) et Umberto Eco (auteur de romans historiques dont le plus extraordinaire - Le Roman de la Rose - pose des questions essentielles sur notre époque et sur le rapport entre les deux types d'histoires, spécialement dans la postface du Roman de la rose ici citée). Une étude systématique de l'approche des fictions historiques sur ces archidupes serait vraiment utile.

Le deuxième aspect qui demanderait un autre ouvrage est commencé ici et exploité à travers une source importante, mais insuffisante. C'est peut-être une histoire de «perdants » selon le point de vue européen et, au moins partiellement, plus glorieux, celle d'une victoire mexicaine. Il faudrait encore un autre livre pour s'interroger sur les perspectives des « Autres ». Car si le livre est basé sur de nombreux documents et récits, ils sont très majoritairement de langue française. Il y aurait donc des études complémentaires, comparatives à écrire non seulement à partir des documents et fictions étrangères (allemandes, espagnoles-mexicaines, et peut-être même maya). Ainsi Juarez, l'ennemi républicain des conservateurs et de l'empire, était métisse maya! Et il serait intéressant d'« entendre » les voix des servantes métisses de Cuernavaca séduites par Maximilien (Goffin, cité p.282). La plus importante serait peut-être à l'intérieur des études coloniales, loin donc du sentimentalisme et romantisme sur ces deux «pauvres individus », mais plutôt comment les Mexicains percevaient ces empereurs franco-belgo-autrichiens.

Alternative francophone

https://journals.library.ualberta.ca/af/index.php/af 
Ainsi si le postfacier mentionne en passant l'absence de sources allemandes dans cette étude (427), il ne mentionne qu'à peine la mexicaine, et se montre eurocentriste et patriarcal dans ses considérations allant jusqu'à utiliser certains mots et certaines idées de manière quelque peu discutable («alors qu'on lui connait maintes liaisons, vénales ou de haut rang — sans parler des indiennes qui lui auraient donné un ou deux fils » (429), sans utiliser de guillemets autour du terme problématique d' « indiennes », et sans mentionner de possibles filles). Ainsi écrit-on des milliers de pages sur ces deux personnages finalement politiquement insignifiants comme le reconnaît le postfacier, « Le peu de traces à long terme qu'ils ont laissées dans l'histoire de leur pays d'adoption » (430), sinon parce qu'ils sont « nobles » et « romantiques », jamais le point de vue mexicain n'est évoqué sinon pour présenter le colonel mexicain Miguel Lopez comme possiblement un traître (430). Mais traître pour qui? Sachant que Querétano est un tournant capital pour la nation mexicaine, mais finalement insignifiant pour ces empires sur leur fin, puisque ces trois empires échouent : l'empire austro-hongrois la même année à Sadowa (1866), l'empire français quelques 4 ans plus tard avec la guerre franco-prusse (1870) et le non-empire belge jusqu'à la conquête brutale du Congo par le père de Charlotte, cet empereur-entrepreneur impitoyable (1885).

Le chapitre cite de nombreux écrivains et historiens dont Goffin qui a une perspective évidemment (en 1937) eurocentriste comme l'atteste la citation suivante : « que fera la jardinière quand l'empereur aura traversé les océans après l'avoir abandonnée dans le grand parc où elle rêve à son dieu blond? Que fera la belle métisse de Cuernavaca qui a donné tant de bonheur à Maximilien ?... » (Goffin cité p. 282)

Certaines sources espagnoles-mexicaines sont citées, mais indirectement comme l'historien mexicain Fernando Iglesias Calderon cité par Reinach (284) et absentes de la bibliographie puisque source indirecte. Heureusement, il y a ces quelques pages consacrées à l'exception intéressante en langue espagnole du grand romancier mexicain Fernando Del Paso et à son roman postmoderne, Noticias del imperio, publié en 1987 et cité en fin de volume (420-421).

Tout ceci n'est point un reproche à l'ouvrage de Bénit, dont je répète qu'il est remarquable et est le fruit d'un travail considérable. C'est pourquoi il ne s'agit pas de le critiquer pour des manques, mais de proposer de nouvelles recherches dont les plus importantes seraient basées sur les nouvelles sources et interprétations autrichiennes et mexicaines,

car on peut se demander en lisant la bibliographie de cette étude si ce sujet n'est pas un fantasme francobelge et français. Tant d'études sur ces deux «perdants » de l'histoire, c'est plus qu'étonnant! En effet, la bibliographie de ce livre contient soixante auteur.e.s dont plus de vingt belges (dont une référence en néerlandais), plus de vingt françaises, trois ou quatre espagnoles (dont pour l'une, l'auteur est belge, mais a publié en espagnol dans une publication espagnole), une en anglais [Haslip qui est américain] et une en italien, mais sans direct rapport avec le sujet, Eco),

Pour conclure, mis à part quelques répétitions (par ex. sur le Père Soria, p. 284 repris p. 291), et un index manquant (malheureusement un défaut assez typiquement francophone), ce livre est indéniablement une œuvre remarquable. Non seulement est-elle bien écrite et remplie d'informations intéressantes, mais elle met en évidence cette fascination problématique du grand public pour les côtés sentimentaux, romantiques, sensationnels. Il commence aussi à poser quelques questions essentielles sur les rapports entre histoire (historiographie) et fiction autour de ces deux « perdants ». On attend avec impatience une ou des réponses en langues étrangères et dans une perspective étrangère, car cette fascination pour ces deux personnages qui avaient tout pour réussir et ont échoué « tragiquement » (ou misérablement ou 
follement) alors que tant d'autres personnages (des milliers de soldats, serviteurs, fermiers mexicains, sans parler de Belges dans les mines, des Austro-Hongrois dans les guerres impériales, de Français dans les prisons napoléoniennes, etc.) sont morts dans la misère à cause de l'arrogance de ces « grands ». André Bénit publie d'ailleurs régulièrement des études pour faire entendre ces voix tu(é)es comme sa citation de l'écrivaine Liliane Wouters les fera entendre à travers les trois servantes de l'empereur citées $(406,417)$. 


\section{SOURCES CITÉES}

LeGoff, Jacques, et Pierre Nora (dir.). Faire de l'histoire, Paris, Gallimard, 1974.

Reyns-Chikuma, Chris. Recension de Charlotte, Princesse de Belgique et impératrice du Mexique (18401927) : Un conte de fée qui tourne au délire par André Bénit, Editions Historic'one, 2017, 219 p. Dalhousie French Studies 111, 2018, pp. 125-126. 